\title{
Effect of Gliotoxin on Human Polymorphonuclear Neutrophils
}

\author{
D.T. Shah, ${ }^{1 *}$ S. Jackman, ${ }^{2}$ J. Engle, ${ }^{2}$ and B. Larsen ${ }^{3}$ \\ ${ }^{1}$ Department of Pathology, Marshall University School of Medicine, Huntington, WV \\ ${ }^{2}$ Department of Microbiology, Immunology \& Molecular Genetics, Marshall University School of \\ Medicine, Huntington, $W V$ \\ ${ }^{3}$ University of Osteopathic Medicine and Health Sciences, Des Moines, IA
}

\begin{abstract}
Objectives: Candida albicans is known to produce gliotoxin, which has several prominent biological effects, including immunosuppression. Interference with host defenses may arise from the effects of this toxin on leukocyte structure and function.

Methods: Flow cytometric analysis revealed that polymorphonuclear leukocytes (PMN) were more sensitive to gliotoxin than were mononuclear cells. Structural and various functional aspects of PMN exposed to gliotoxin were studied.

Results: Gliotoxin at $(1 \mu \mathrm{g} / \mathrm{mL})$ did not affect the viability but did diminish PMN chemotaxis and reduced their ability to ingest particles. Other functional aberrations included decreased nitroblue tetrazolium dye reduction, decreased superoxide production, and release of lactoferrin suggesting by degranulation. Gliotoxin also affected the ability of PMN to kill Escherichia coli.

Conclusions: This study suggests a previously unrecognized potential virulence factor of $C$. albicans that could contribute to persistence of yeast colonization or recurrence of symptomatic infection through diminished host resistance. Infect. Dis. Obstet. Gynecol. 6:168-175, 1998.

(C) 1998 Wiley-Liss, Inc.
\end{abstract}

KEY WORDS

immunosuppression; virulence factors; phagocytosis

M ucocutaneous candidiasis is a common problem among immunocompromised patients, including those with acquired immunodeficiency syndrome (AIDS), but it can also occur in individuals without known immune defects. Virulence attributes of Candida albicans, particularly those that may suppress the immune system, remain incompletely investigated. Several reports describe products of Candida that may contribute to its virulence, including a protease ${ }^{1}$ and cell wall mannans that have been reported to interfere with neutrophil function. ${ }^{2}$ In addition, a crude product of candidal hyphae was shown to prevent normal superoxide production by stimulated neutrophils. ${ }^{3}$
A new dimension was added to study Candida virulence when we found that clinical isolates of this organism produced gliotoxin, ${ }^{4}$ an epipolythiodioxopiperazine mycotoxin. This class of compounds is known for various biological actions, including antibacterial, antiviral, and immunosuppressive activities. ${ }^{5}$ We subsequently analyzed vaginal samples of three women severely symptomatic for vaginal candidiasis and found that they contained significant levels of gliotoxin. ${ }^{6}$ We reasoned that in vivo production of gliotoxin by $G$. albicans may contribute to its virulence and chronicity of disease by circumventing local host defenses. In mucocutaneous candidiasis, polymor-

Grant Sponsor: Johnson \& Johnson Corporate Office of Science and Technology.

*Correspondence to: D.T. Shah, PhD, Department of Pathology, Marshall University School of Medicine, Huntington, WV. 
phonuclear leukocytes (PMN) seemed to be crucial as a prominent defense mechanism. This study was undertaken to evaluate effect of gliotoxin on human PMN functions.

\section{MATERIAL AND METHODS}

Crystalline gliotoxin was purchased from Sigma Chemical Company (St. Louis, MO). Purchased gliotoxin was found identical to the toxin produced by clinical isolates of $G$. albicans. ${ }^{7}$ Stock solutions were prepared in methanol and further diluted in RPMI or phosphate-buffered saline with glucose (PBSG) to attain the desired final concentrations.

Preparation of whole heparinized blood for flow cytometry used blood drawn from healthy volunteers. To $10 \mathrm{~mL}$ of whole blood, $14 \mathrm{~mL}$ of lysing reagent $\left(\mathrm{NH}_{4} \mathrm{Cl}, 8 \mathrm{~g} / \mathrm{L} ; \mathrm{KHCO}_{3}, 1.0 \mathrm{~g} / \mathrm{L}\right.$, tetrasodium EDTA, $37.0 \mathrm{mg} / \mathrm{mL}, \mathrm{pH} 7.3$ ) was added, mixed for 3 to 5 minutes, and centrifuged for 30 minutes at $300 \mathrm{~g}$ at room temperature. The cell pellet was washed twice with phosphate-buffered saline (PBS) and resuspended in $1 \mathrm{~mL}$ of PBS.

PMN preparations were obtained by gradient centrifugation of whole blood through Mono-Poly Resolving Medium (Flow Laboratories, McLean, VA) according to the manufacturer's directions. The PMN layer was aspirated and delivered into tubes containing calcium-free PBSG. Cell viability (>95\%) was confirmed with trypan blue dye exclusion, and counts were made in a hemacytometer. Dilutions of PMN were made in PBSG with $0.001 \mathrm{M} \mathrm{CaCl}_{2}$ and $0.1 \%$ gelatin (Sigma).

Flow cytometry employed a FACscan instrument (Beckon Dickinson Immunocytometry Systems, Mountain View, CA). All analyses were performed on Leucogate R (Beckon Dickinson, San Jose, CA) antibody treated cells. Each analysis consisted of a minimum of 5,000 gated events. Histograms were contoured and visually inspected for the presence of distinct populations Gates were then chosen to incorporate all events associated with distinct populations. These gates were used to analyze all subsequent data sets. The effect of gliotoxin on leukocytes was initially investigated by exposing blood cells to high doses of gliotoxin (0.5$500 \mu \mathrm{g} / \mathrm{mL})$ in PBSG $(\mathrm{pH} 7.3)$ at a cell concentration of $1 \times 10^{6}$ leukocytes $/ \mathrm{mL}$ at $37^{\circ} \mathrm{C}$ for $30 \mathrm{~min}$ utes. At the end of the incubation period, $5 \mu \mathrm{L}$ of Leucogate antibody (Beckon Dickinson) was added, and the labeling proceeded in the dark at room temperature for 15 minutes, followed by flow cytometric analysis.

Cell viability after gliotoxin exposure was demonstrated both by trypan blue dye exclusion and by flow cytometry of propidium iodide treated cells. Leukocytes $\left(1 \times 10^{6}\right.$ leukocytes $\left./ \mathrm{mL}\right)$ were incubated with gliotoxin diluted in PBSG or RPMI at $37^{\circ} \mathrm{C}$. Cells were exposed to $0.5-500 \mu \mathrm{g} / \mathrm{mL}$ of gliotoxin, and samples were taken at different time intervals ranging from 15 to 90 minutes. Samples were further mixed with $5 \mathrm{~mL}$ of $0.0005 \%$ propidium iodide (Sigma) and analyzed by flow cytometry.

After determining the gliotoxin concentration (1 $\mu \mathrm{g} / \mathrm{mL}$ ), PMN chemotaxis was investigated in modified Boyden chambers. Gliotoxin-treated PMN (1 $\mu \mathrm{g}$ gliotoxin, $1 \times 10^{6} \mathrm{PMN} / \mathrm{mL}$ ) were activated with LPS-treated human serum $(0.1 \mathrm{mg}$ of LPS to $1 \mathrm{~mL}$ of human serum was incubated for 60 minutes at $37^{\circ} \mathrm{C}$ ). At the end of 30 minutes of incubation, the pattern of migration of PMN across a polycarbonate filter ( $3 \mu \mathrm{m}$ pore size) was evaluated after Giemsa staining by light microscopy. Controls consisted of PMN incubated without gliotoxin.

Particle ingestion by PMN was evaluated by uptake of fluorescein-conjugated styrene beads (Flow Cytometry Standards Corp., NC). The PMN $\left(1 \times 10^{6} / \mathrm{mL}\right)$ were incubated for 15 minutes with 1 $\mu \mathrm{g} / \mathrm{mL}$ gliotoxin and mixed with beads to achieve a particle-to-cell ratio of 100:1. The mixture was gently mixed on a rotating wheel, and aliquots were removed at timed intervals. Aliquots were centrifuged in medium containing $2 \% \mathrm{BSA}$, and the cells recovered were resuspended in MEM with $20 \mathrm{mM}$ 2-N-morpholine propane sulfonic acid with $10 \%$ fetal bovine serum. The fluorescence due to ingested beads was measured by flow cytometry.

Nitroblue tetrazolium (NBT) reduction was used to measure respiratory burst activity. The PMN $\left(1 \times 10^{6}\right.$ cells $\left./ \mathrm{mL}\right)$ were pretreated with 1 $\mu \mathrm{g} / \mathrm{mL}$ gliotoxin (control cells remained untreated) with stimulant consisting of the supernatant from zymosan (Sigma) treated serum. One tenth of a milliliter of NBT was added to the mixture and incubated for 10-20 minutes, and the development of color was monitored spectrophotometrically at 570 with 630 used as the reference wavelength.

Production of superoxide anion by gliotoxintreated or untreated cells was determined spectro- 
A

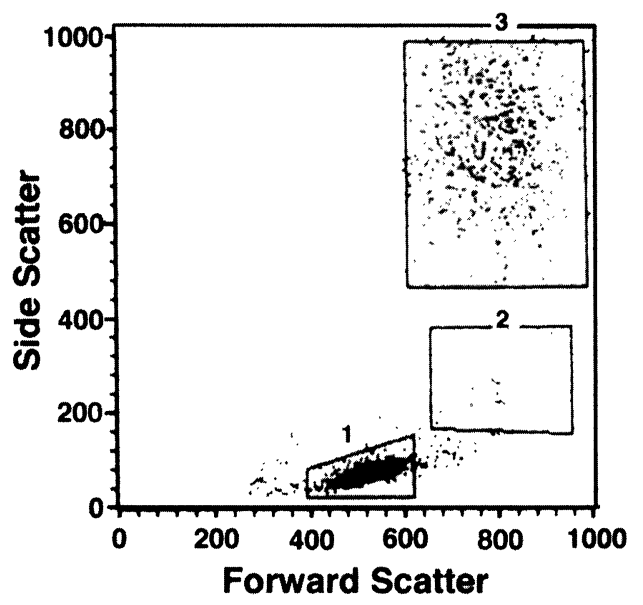

B

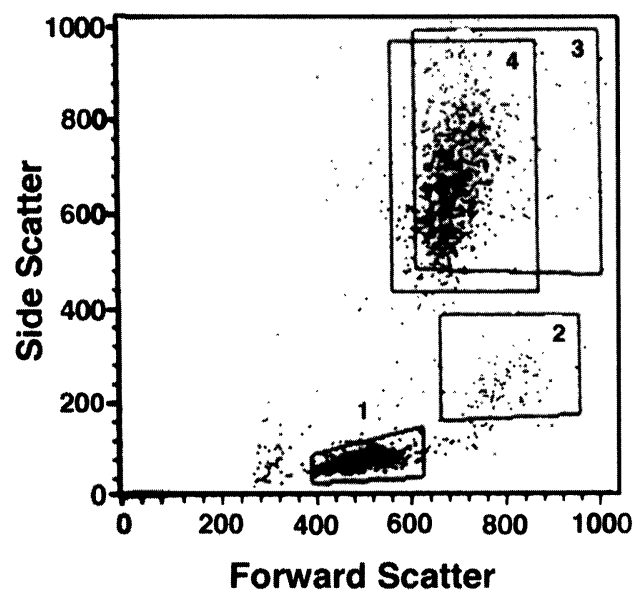

Fig. I. Flow cytometric analysis of human blood leukocytes. Flow cytometric analysis of peripheral blood collected from healthy donors was performed in a FACscan flow cytometer. (A) Windows I, 2, and 3 represent lymphocytes, monocytes, and PMN. The leukocyte populations are separated on the basis of forward vs side scatter. (B) Human leukocytes exposed to $500 \mu \mathrm{g} / \mathrm{mL}$-concentration of gliotoxin for 30 minutes showed a shift in PMN population from right to left on the forward scatter axis and downward on the side scatter scale, consistent with these cells becoming smaller and less granular.

photometrically by reduction of cytochrome $\mathrm{C}$ as described elsewhere. ${ }^{8}$ Experiments were conducted in sets of three tubes in which phorbol myristate acetate (PMA, $10 \mu \mathrm{L} / 5 \mathrm{~mL}$ ) was used as an activator for PMN superoxide production. The first tube was a cell-free control that showed baseline superoxide production from PMA, the second tube contained superoxide dismutase $(2 \mu \mathrm{g})$ with cells and PMA to establish assay specificity, and the third tube contained cells and PMA and was the positive test. Cell concentration was $1 \times 10^{7} \mathrm{PMN}$ in $1 \mathrm{~mL}$ of HEPES (N-2-Hydroxyethylpiperazine-N2-ethenesulfonic acid) buffered medium (HBM) and $0.4 \mathrm{~mL}$ of cytochrome $\mathrm{C}(18.6 \mathrm{mg} / \mathrm{mL})$. Tests were performed in triplicate comparing cells treated with $1 \mu \mathrm{g} / \mathrm{mL}$ gliotoxin with untreated cells. After mixing the compounds, all tubes were incubated at $37^{\circ} \mathrm{C}$ for 15 -minute timed intervals after which cells were removed by centrifugation and the absorbance of the supernatant fluid was read at $530 \mathrm{~nm}$. Superoxide was calculated as follows: $\mathrm{nm}$ superoxide $/ 10^{6} \mathrm{PMN} / \mathrm{h}=\delta \mathrm{Abs} \times 10^{3} / 21$ where $\delta A b s$ means the difference in absorbance between the samples with and without superoxide dismutase after subtracting time zero values from each.

Intracellular killing of Escherichia coli by PMN was determined by suspending $2.5 \times 10^{6} \mathrm{PMN}$ in 0.1 $\mathrm{mL}$ of fetal calf serum and $0.3 \mathrm{~mL}$ HBS and con- ditioning them for 5 minutes at $37^{\circ} \mathrm{C}$. Subsequently, $2 \times 10^{7}$ E. coli contained in $0.1 \mathrm{~mL}$ of HBS were added to each well. Aliquots were removed at timed intervals and centrifuged at $150 \mathrm{~g}$ for $5 \mathrm{~min}$ utes, and the supernatant fluid was removed. The supernatant-containing bacteria that had not been ingested $(100 \mu \mathrm{L})$ were added to $9.9 \mathrm{~mL}$ of sterile water, and dilutions were plated on nutrient agar. The pellet that contained the PMN was suspended in sterile water to lyse the cells and release viable intracellular bacteria, which were enumerated by viable plate count.

\section{RESULTS}

The effect of gliotoxin on the various types of leukocytes in peripheral blood was tested by incubating $500 \mu \mathrm{g}$ gliotoxin per $\mathrm{mL}$ of whole blood lysate for 30 minutes at $37^{\circ} \mathrm{C}$. As illustrated by Figure 1, the granulocyte population appeared more affected (decreased forward scatter and decreased side scatter) than either the mononuclear cell population or the lymphocytes population. Thus, it appeared that gliotoxin may have caused a decrease in granulocyte size and granularity. To determine if the gliotoxin-treated leukocytes were dying, viability was measured by propidium iodide uptake. Gliotoxin $\left(0.5-500 \mu \mathrm{g} / \mathrm{mL}\right.$ for $15-90$ minutes at $\left.37^{\circ} \mathrm{C}\right) \mathrm{did}$ not appear to damage lymphocytes, whereas granulocytes and monocytes were stained with propidium 


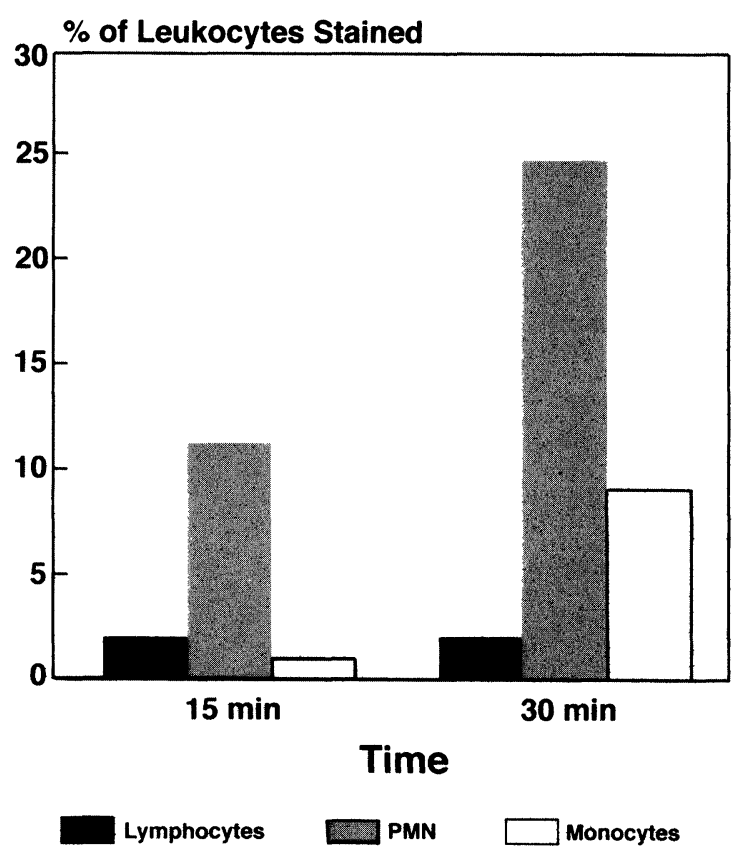

Fig. 2. Gliotoxin effect on leukocyte viability. Human leukocytes were exposed to $500 \mu \mathrm{g} / \mathrm{mL}$ of gliotoxin for 15 and 30 minutes. Viability was measured by propidium iodide uptake. PMN were the most affected when compared with lymphocytes and monocyte populations of the peripheral blood.

iodide (Figure 2). After 30 minutes of exposure to gliotoxin, $24 \%$ of granulocytes were stained with propidium iodide and $9 \%$ of monocytes were stained.

Because the granulocyte population, which consists mainly of PMN, appeared to be the most susceptible to gliotoxin, subsequent studies focused mainly on PMN that were isolated from healthy donors by Ficoll-Hypaque density gradient centrifugation. Isolated PMN exposed to gliotoxin $\left(500 \mu \mathrm{g} / \mathrm{mL}\right.$ for $15-90$ minutes at $37^{\circ} \mathrm{C}$ ) showed an increase in propidium iodide uptake that was both gliotoxin dose-dependent and time-dependent, as shown by Figure 3 . These results were corroborated by trypan blue dye exclusion that showed that minimum cytocidal gliotoxin concentration appeared to be greater than $1 \mu \mathrm{g} / \mathrm{mL}$.

Gliotoxin concentration $(1 \mu \mathrm{g} / \mathrm{mL})$ was selected, which did not have a cytocidal effect on PMN. To systematically determine if gliotoxin could cause functional aberration in PMN, several aspects of phagocytic function in gliotoxin-treated $(1 \mu \mathrm{g} / \mathrm{mL})$ and control PMN were investigated. The vigor of chemotaxis in gliotoxin-treated PMN was evaluated in Boyden chambers. Gliotoxin-treated $(1 \mu \mathrm{g} /$

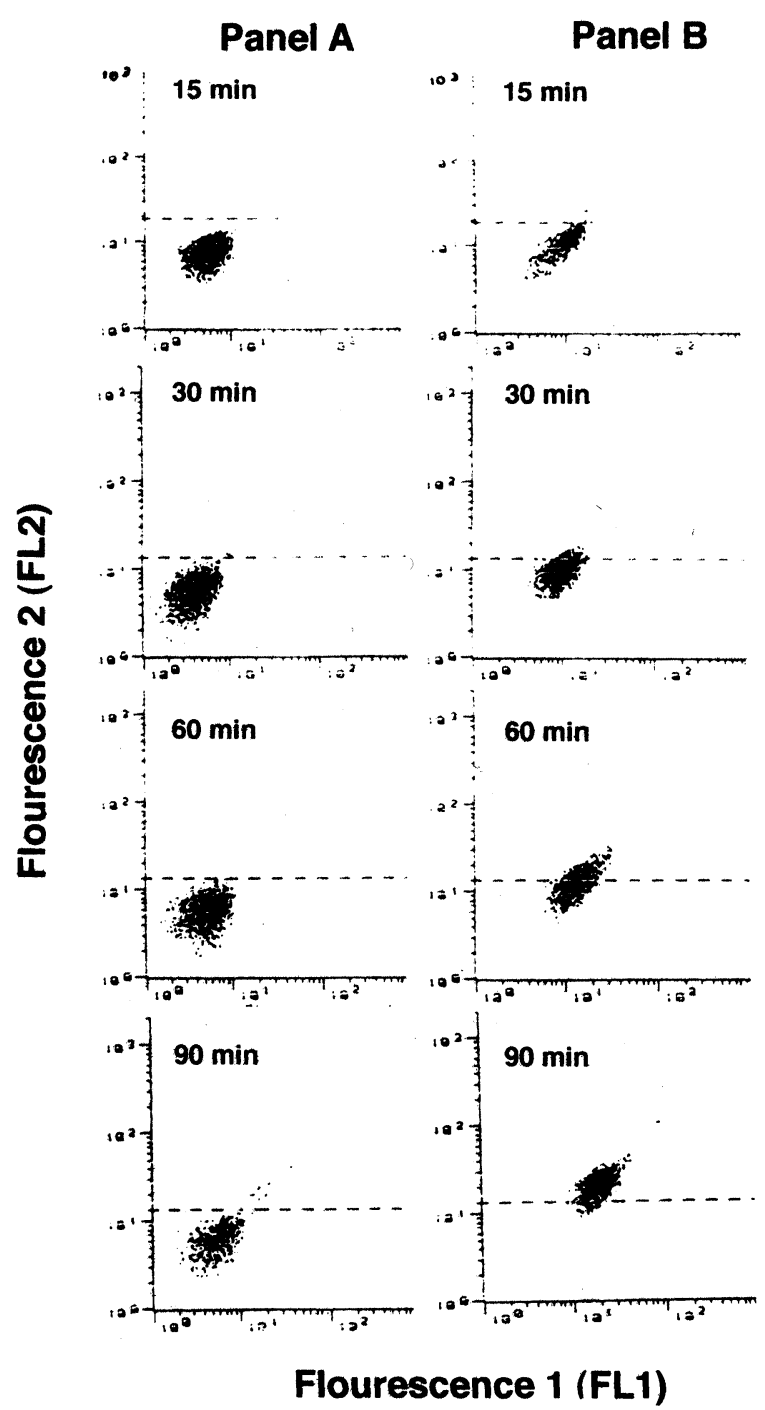

Fig. 3. Propidium iodide uptake measures by FL2 fluorescence. FL2 fluorescence (due to PI uptake) was increased by increasing the incubation time of gliotoxin-treated $(500 \mu \mathrm{g} /$ $\mathrm{mL}$ ) PMN. Panel A represents the unintoxicated PMN, which do not show significant increases in FL2 fluorescence. As the incubation time increases, more PMN are seen in the FL2 region of panel B, which represents gliotoxin-treated PMN.

mL) PMN migrated less effectively across the membranes than untreated PMN, as shown in Figures $4 \mathrm{~A}$ and $4 \mathrm{~B}$.

Phagocytic function depends in part on the ability of PMN to attach to and engulf particles. The effect of gliotoxin on these aspects of phagocytosis was determined by monitoring the uptake of fluorescent styrene beads and measured by flow cytometry. As shown in Figure 5, 40\% of untreated PMN engulfed particles within 90 minutes, 

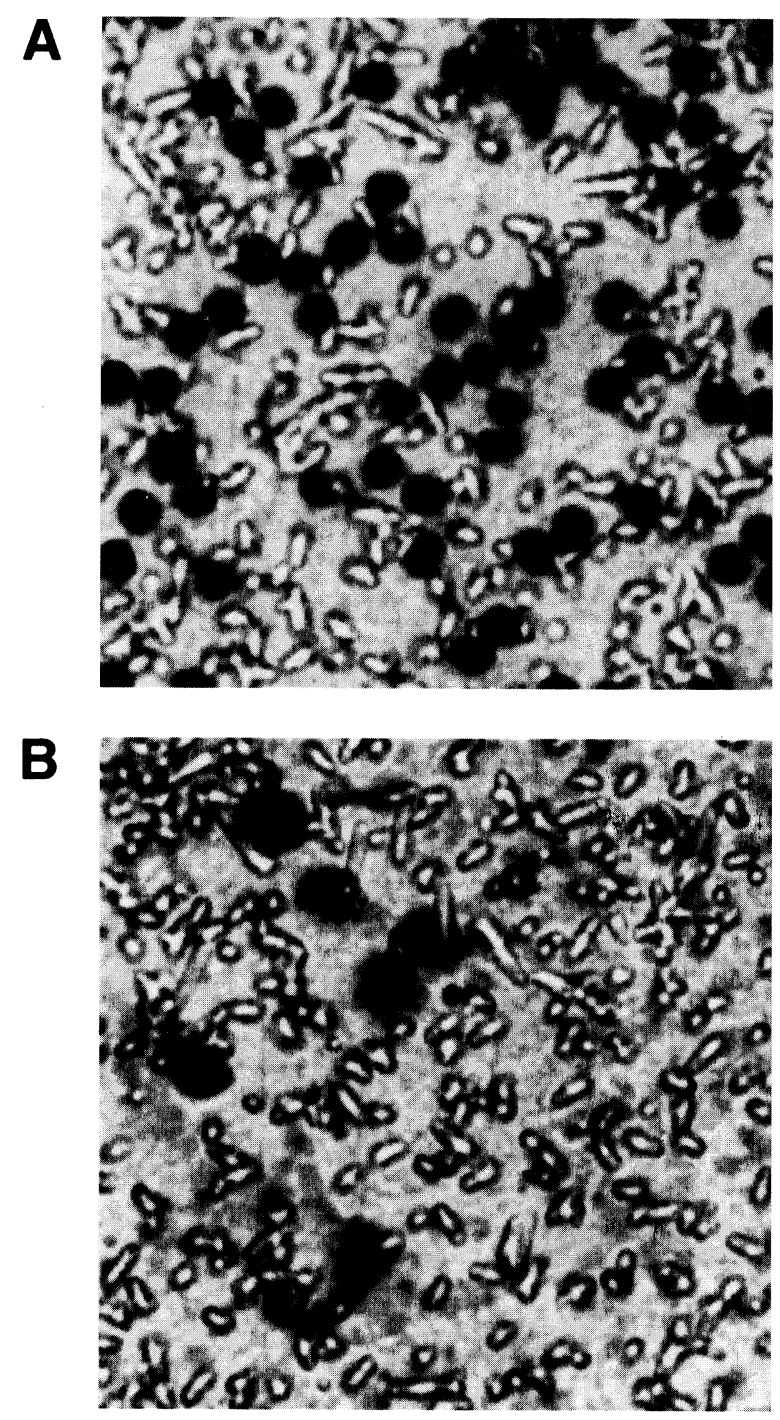

Fig. 4. (A) Control (untreated) PMN migration. Migration pattern of untreated PMN across the polycarbonated membrane was observed by staining the filter with Giemsa. The stained membrane was placed on a glass slide with an oil drop and a coverslip and observed under light microscopy. Clear channels represent the pores present in the membrane. (B) Migration of gliotoxin-treated (I $\mu \mathrm{g} / \mathrm{mL}$ ) PMN. PMN crossing the polycarbonate membranes were quantitated and plotted for each of the donors participating in this study.

whereas only $13 \%$ of cells treated with $1 \mu \mathrm{g} / \mathrm{mL}$ of gliotoxin/mL for 90 minutes ingested fluorescent beads.

Actively phagocytic active cells increase their oxidative metabolism resulting in the generation of reactive oxygen species such as superoxide. The increased reducing capacity of phagocytically active leukocytes may be measured by nitroblue tetrazolium dye reduction. Figure 6 shows that

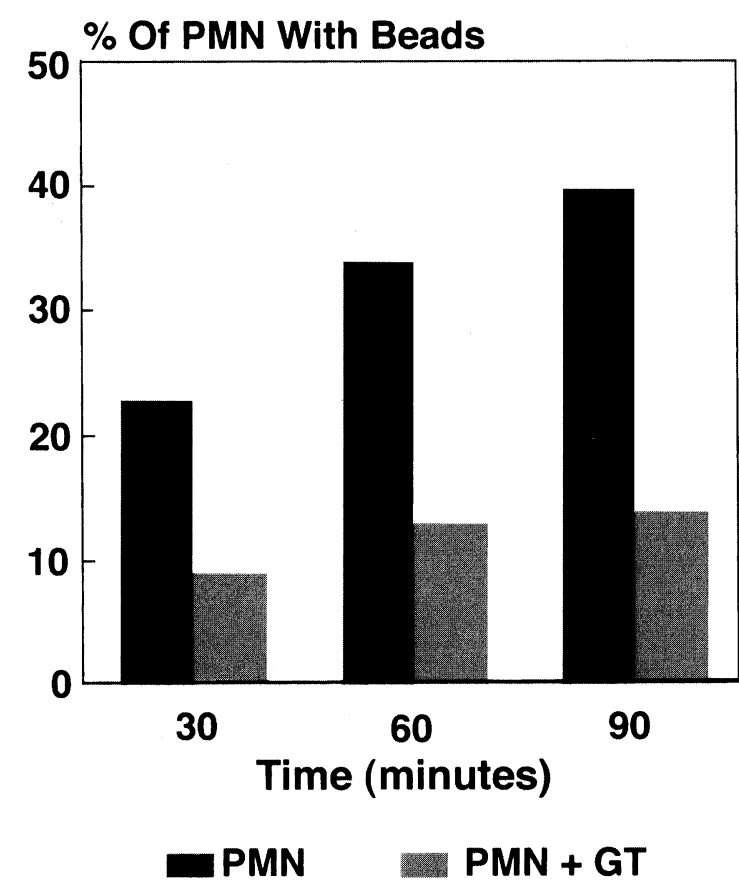

Fig. 5. Particle engulfment by gliotoxin-treated $(1 \mu g / m L)$ PMN. A PMN suspension (gliotoxin-treated [GT] and -untreated) was adjusted to $1 \times 10^{6}$ cells $/ \mathrm{mL}$. The cells were co-incubated with fluorescein-conjugated beads $\left(1 \times 10^{8}\right.$ cells $/ \mathrm{mL}$ ) at $37^{\circ} \mathrm{C}$ for 30 to 90 minutes. The effect of gliotoxin on particle uptake by PMN was investigated by flow cytometry.

complement-stimulated gliotoxin-treated $(1 \mu \mathrm{g} / \mathrm{mL}$ of gliotoxin for 10 minutes at $37^{\circ} \mathrm{C}$ ) PMN had diminished capacity for dye reduction compared with stimulated control PMN.

The antibacterial mediator, superoxide anion, was decreased when cells were pretreated with gliotoxin, compared with untreated cells as shown by Figure 7.

The final aspect of neutrophilic cell function studied was intracellular bacterial killing. Viable $E$. coli was used as the test organism, and PMN pretreated with gliotoxin or untreated control cells were allowed to ingest and kill the test bacterium. Whereas control PMN inactivated $79 \%$ of $E$. coli within the first 30 minutes after mixing bacteria and phagocytic cells, gliotoxin-treated cells failed to kill any E. coli. By 90 minutes, control PMN had killed $93 \%$ of the bacteria, and the gliotoxintreated PMN killed $79 \%$ of the bacterial challenge (Figure 8).

\section{DISCUSSION}

Although the literature regarding the pathogenic potential of Candida is extensive, our understand- 


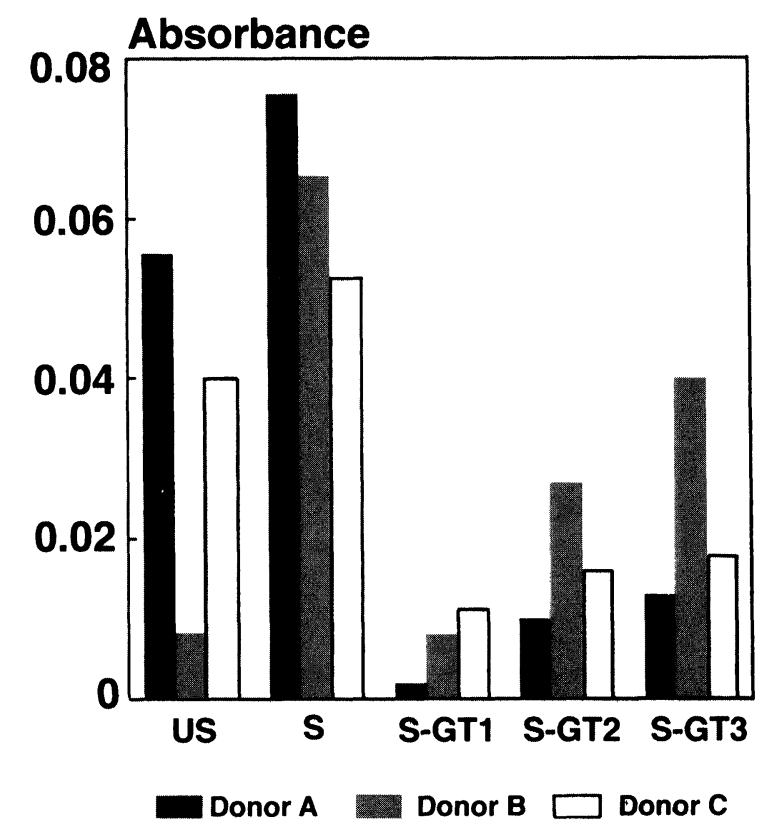

Fig. 6. Metabolic stimulation of gliotoxin-treated (I $\mu \mathrm{g} /$ $\mathrm{mL}$ ) PMN. NBT reduction by unstimulated (US) PMN, unstimulated gliotoxin-treated PMN, zymosan-stimulated (S) PMN, and zymosan-stimulated gliotoxin-treated (S-GT) PMN was measured spectrophotometrically at $570 \mathrm{~nm}$ wavelength with a reference wavelength of $630 \mathrm{~nm}$.

ing of specific virulence factors and their role in pathogenesis remains limited. In mucocutaneous candidiasis, PMN seemed to be crucial as a prominent defense mechanism. ${ }^{9}$ Patients with few neutrophils or dysfunctional phagocytes are more susceptible to disease with Candida than individuals with normal neutrophil function.

Interestingly, the results of this study suggest that gliotoxin is more deleterious to PMN than mononuclear cells or lymphocytes from peripheral blood, as evidenced by propidium iodide staining. This cytocidal effect, however, employed concentrations of gliotoxin that probably would not occur in vivo. ${ }^{10}$

Both because of the importance of PMN in candidal infections and because of their apparent susceptibility to gliotoxin, we sought to identify functional alterations in gliotoxin-treated PMN. Our approach involved examining the various elements of the phagocytic response, including directed migration, particle engulfment, respiratory increment, and bactericidal effects. Because of the opportunity for the interaction between mucosal leukocytes and mucosal organisms, the inhibition of phagocytosis could play a role in the persistence or recur- rence of candidiasis. As shown in the present study, all aspects of phagocytic function were altered to some degree by incubation with gliotoxin.

The possibility that gliotoxin may have a role in vaginal infection is dependent on whether the organism can actually produce the toxin in vivo. In previous studies we found that gliotoxin can be produced by Candida inoculated into fluid obtained on vaginal swabs from patients. ${ }^{7} \mathrm{We}$ also showed that women with yeast vaginitis had detectable levels of gliotoxin, and these levels were sufficient to cause the altered phagocytic function as reported in the present study. Since we were able to isolate the gliotoxin from the yeast vaginitis patients, we predict that it is not degraded but may be neutralized with the use of antimycotic agent.

While this investigation suggests a potential role for gliotoxin in mucocutaneous candidiasis, the mechanism of the deleterious effects on phagocytic function was not directly investigated. Several observations may be enlightening with regard to the potential role of gliotoxin in vivo. Engulfment of particles requires ligation of the particles to the phagocyte followed by membrane invagination. The apparent structural changes in leukocytes as shown by flow cytometry in concert with microscopic observation of cell blebbing (data not shown) suggest that membrane changes that decrease the effectiveness of particle ingestion may occur in response to gliotoxin exposure. Sporidesmin, another fungal toxin that is a chemical congener of gliotoxin, has been reported to cause disappearance of liver cell microvilli, ${ }^{11}$ further suggesting membrane perturbation among compounds of this type. Clearly, more definitive studies in this area are warranted.

Several investigators have suggested mechanisms whereby gliotoxin exerts its immunosuppressive effects, and these theories provide a good basis for a more mechanistic study of biological activity. For example, the disulfide component of gliotoxin could cross link membrane receptors. Petty $^{12}$ indicated that there may be a disulfide link between $\mathrm{Fc}$ receptors for antibody, and the interpolation of gliotoxin between these receptors could alter the ability to trigger appropriate phagocytic activity.

Moreover, this study suggested that while gliotoxin is able to reduce the respiratory burst activity of stimulated PMN, the toxin alone in the absence 
A

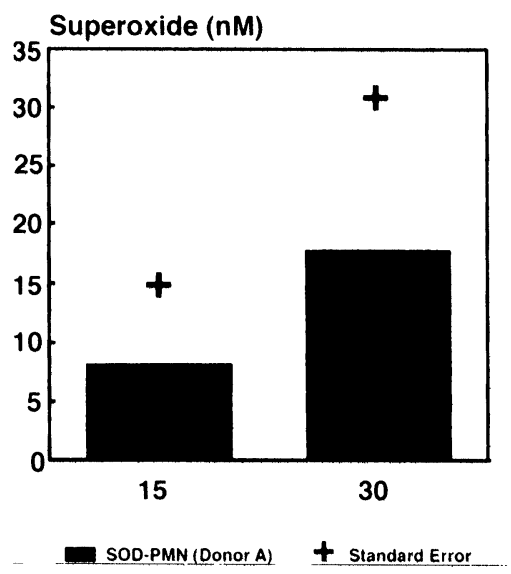

B

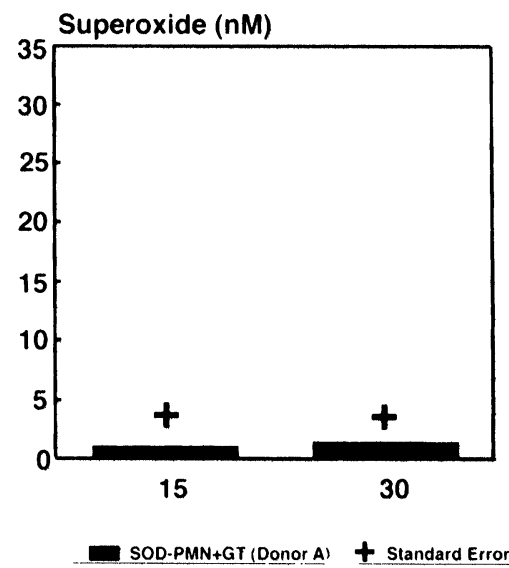

Fig. 7. Superoxide production by gliotoxin-treated $(1 \mu \mathrm{g} / \mathrm{mL})$ PMN. Gliotoxin-treated $(\mathbf{A})$ and untreated (B) cells were stimulated with phorbol myristate. Superoxide was measured spectrophotometrically $(570 \mathrm{~nm})$ by cytochrome $C$ reduction in the presence and absence of superoxide dismutase. This study was conducted in triplicate with PMN collected from two healthy human donors.

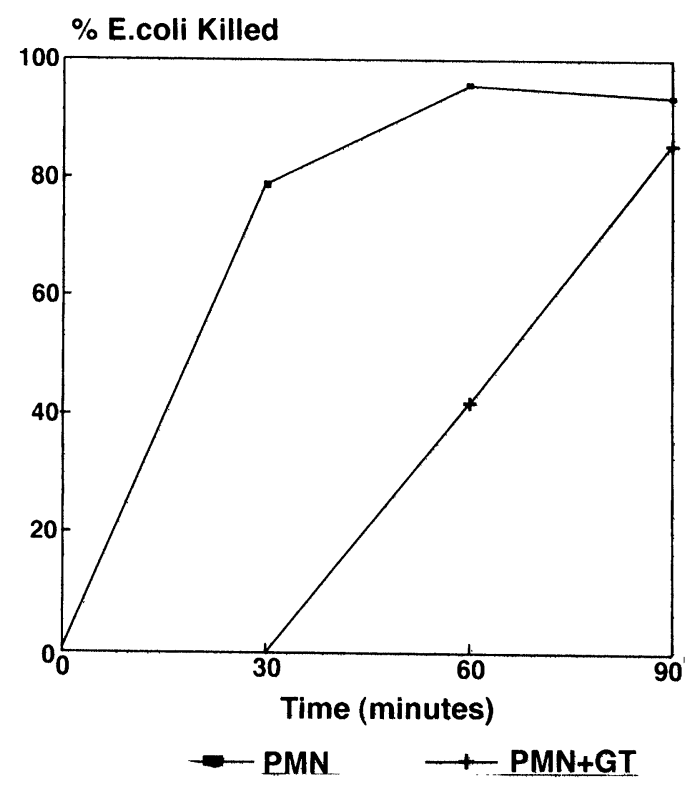

Fig. 8. Intracellular killing of $E$. coli by gliotoxin-treated (I $\mu \mathrm{g} / \mathrm{mL}$ ) PMN. Intracellular killing of $E$. coli by gliotoxintreated (GT) and untreated PMN was determined by differential centrifugation technique.

of a stimulant caused a relatively slight increment in NBT reduction (Fig. 6), indicating that inappropriate or gratuitous receptor triggering may result from interaction of PMN with gliotoxin. Such possibility was further undiscovered by the finding that gliotoxin-treated PMN released lactoferrin (data not shown), implying premature degranulation.

A final potential mechanism of gliotoxin action is induction of apoptosis, a cell process that is not inconsistent with the above-mentioned mechanisms of cytotoxicity. The observation of membrane changes noted above coupled with reports in the literature that attribute apoptotic effects to gliotoxin underscore this as a potential mechanism of action.

The present study indicates that there may be hitherto unrecognized reasons why vaginal yeast infection in some women can be chronic or recurrent and provides a new factor that deserves consideration in future studies of this condition.

\section{ACKNOWLEDGMENT}

This work was supported in part by a focused giving grant from the Johnson \& Johnson Corporate Office of Science and Technology.

\section{REFERENCES}

1. Borg M, Ruchel R: Expression of extracellular acid proteinase by proteolytic Candida spp. during experimental infection or the oral mucosa. Infect Immun 56: 626-631, 1988.

2. Wright CD, Herron MJ, Gray GR, Holmes B, Nelson RD: Influence of yeast mannan on human neutrophil functions: Inhibition of release of myeloperoxidase related to carbohydrate binding property of the enzyme. Infect Immun 32:731-738, 1981.

4. Shah DT, Larsen B: Clinical isolates of yeast produce a gliotoxin-like substance. Mycopathologia 116: 203-220, 1991.

5. Danley DL, Polakoff J: Rapid killing of monocytes in 
vitro by Candida albicans yeast cells. Infect Immun 51: 307-313 1986.

6. Shah DT, Glover DD, Larsen B: In situ mycotoxin production by Candida albicans in women with vaginitis. Gynecol Obstet Invest 39:67-69, 1995.

7. Shah DT, Larsen B: Identity of Candida albicans toxin and its production in vaginal secretions. Med Sci Res 20:353-355, 1992.

8. Pick E, Mizel D: Rapid microassay for the measurement of the superoxide and hydrogen peroxide modulation by monocytes in culture using an automatic enzyme assay reader. J Immunol Methods 46:211-226, 1981.

9. Leibovici V, Evron R, Matzner Y: The effect of Tricho- phytin and Candidin on neutrophil chemotaxis. Mycoses 31:137-142, 1988.

10. Mullbacher A, Waring P, Eichner RD: Identification of an agent in culture of Aspergillus fumigatus displaying antiphagocytic and immunomodulating activity in vitro. J Gen Microbiol 131:1251-1258, 1985.

11. Cordiner JE, Jordan TW: Inhibition by sporidesmin of hepatic bile acid transport. Biochem J 212:197-204, 1983.

12. Petty HR: A sulfhydral redox model of antibodydependent phagocytosis. Mol Immunol 22:1001-1003, 1985. 


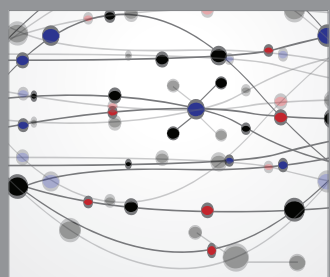

The Scientific World Journal
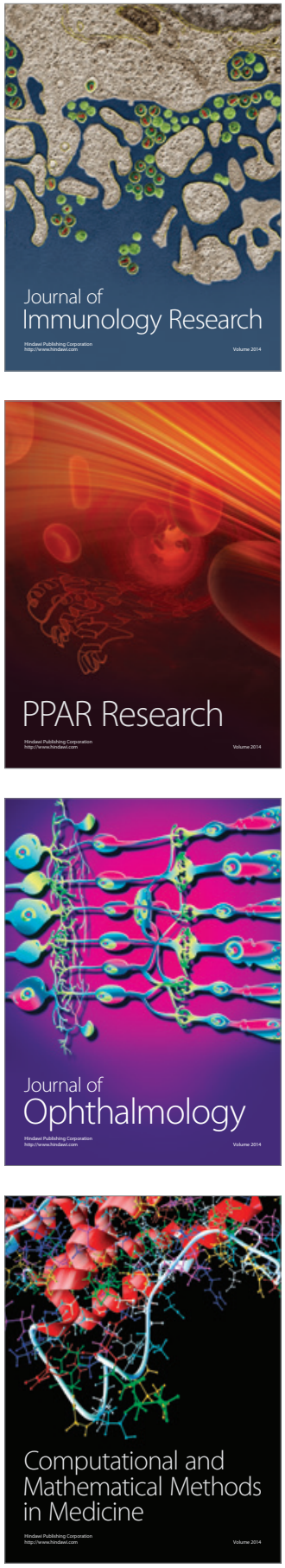

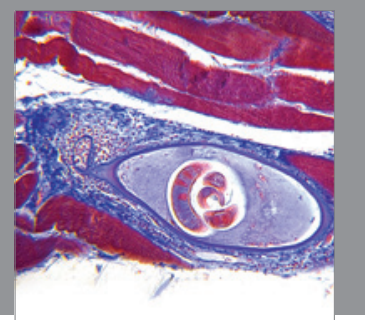

Gastroenterology

Research and Practice
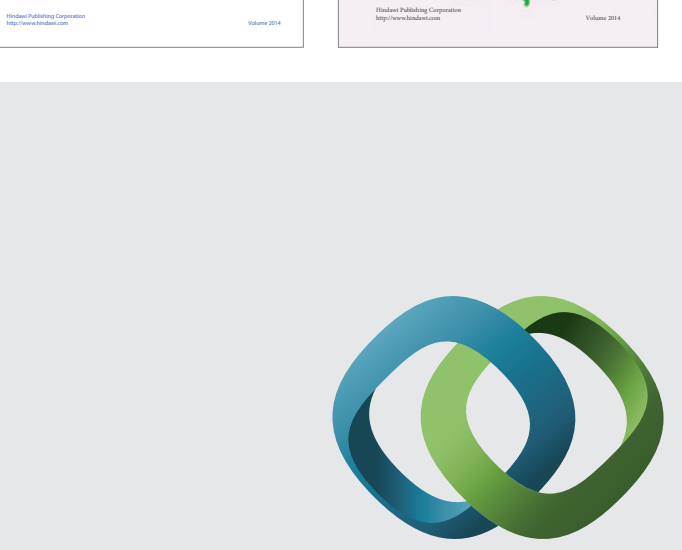

\section{Hindawi}

Submit your manuscripts at

http://www.hindawi.com
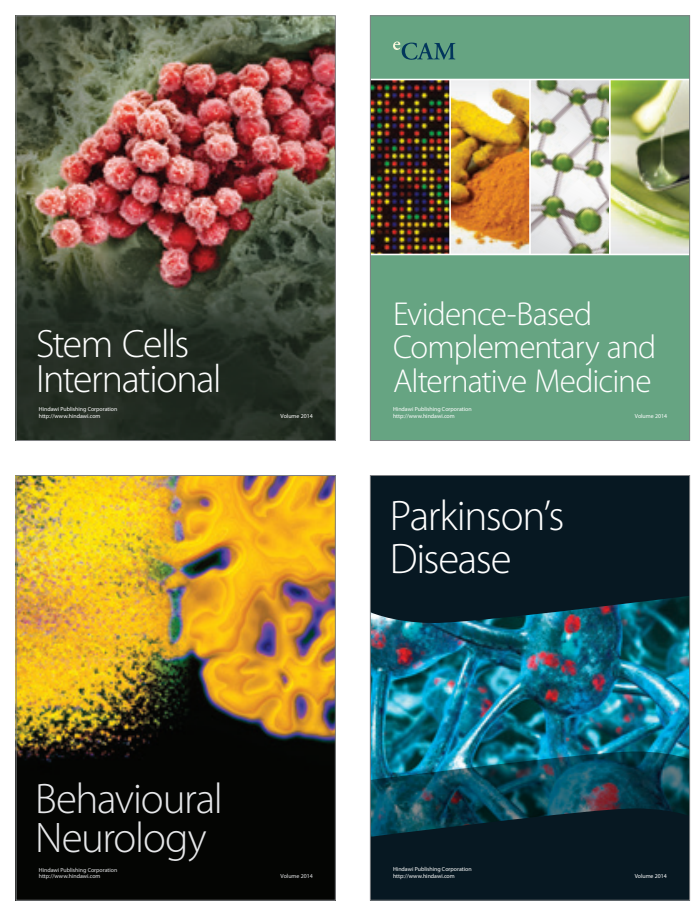

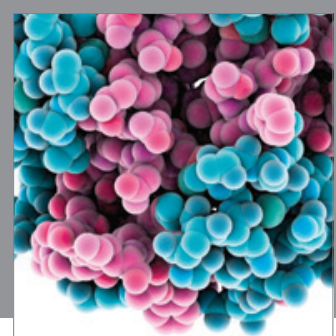

Journal of
Diabetes Research

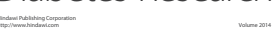

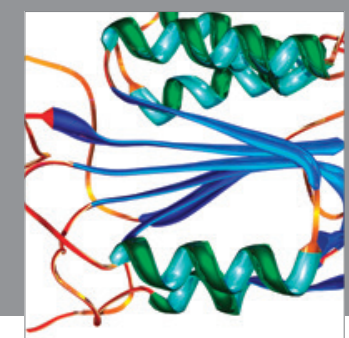

Disease Markers
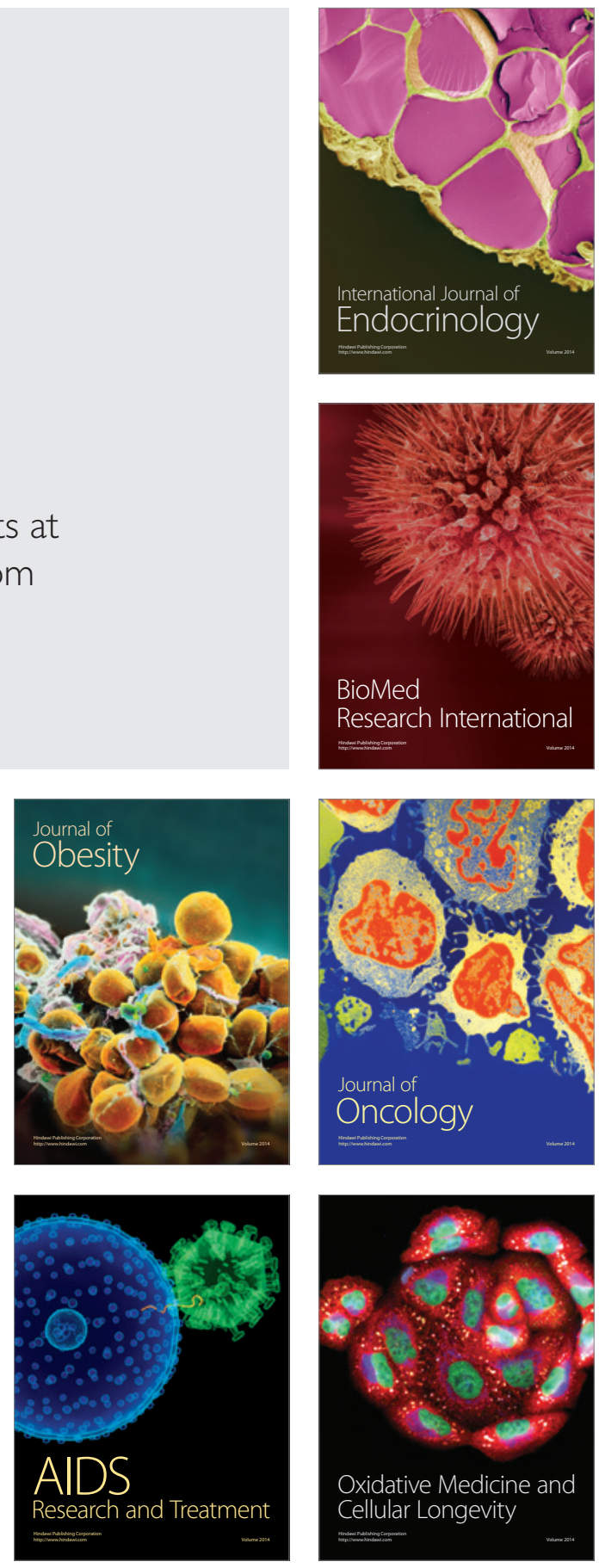